\title{
Impact of Open Vs Laparoscopic Kidney Harvesting and Routine Use of Double J Stent on Longterm Outcome of Ureteral Complications in Renal Transplants
}

\author{
Aneesh Srivastava ${ }^{\mathrm{a}, \mathrm{b}}$, Jatinder Kumar ${ }^{\mathrm{a}}$, Rohit Upadhyaya ${ }^{\mathrm{a}}$, M S Ansari ${ }^{\mathrm{a}}$, Rakesh Kapoor ${ }^{\mathrm{a}}$
}

\begin{abstract}
Background: Ureter related complications are an important cause of morbidity in renal transplant patients. Herein we present our experience and management of such complications. The impact of laproscopic live donor nephrectomy on ureter related complications was also analyzed.
\end{abstract}

Methods: Our practice of vesico-ureteral anastomosis has evolved as phase I from 1989 to 1993 when stent was put only when felt necessary, phase II from January 1994 to April 1995, when stent placement was randomized and phase III from May 1995 to December 2010, where all anastomosis were stented. Kidney was removed by open method till 1998 and laparoscopic nephrectomy (left side) was started from 2002 onwards.

Results: Incidence of ureteral leak without DJ stent was $6.1 \%$ and $0.4 \%$ with stent. Overall incidence of ureteral stenosis was $0.8 \%$. Incidence of stenosis in stented and non-stented group was $0.8 \%$ and $2.3 \%$ respectively. Treatment offered for ureteral stenosis was percutaneous nephrostomy and antegrade stent. Ureteral leak was present in $0.3 \%$ and $1.5 \%$ in open and laproscopic method respectively and ureteral stenosis in $0.9 \%$ and $0.75 \%$ respectively.

Conclusions: Two major ureter related complications were leak and stenosis and three-fourth of them resolved by minimal invasion. Major surgical revision was needed in around one-fourth of cases. Stent use helps in reducing incidence of ureteral leak, but doesn't seem to have any impact on ureteral stenosis. Laparoscopic technique doesn't seem to have any adverse effect on ureter-related complications.

Manuscript accepted for publication June 12, 2012

${ }^{a}$ Department of Urology and Renal Transplantation, Sanjay Gandhi Postgraduate Institute of Medical Sciences, India

${ }^{\mathrm{b}}$ Corresponding author: Aneesh Srivastava, Department of Urology and

Renal transplantation, Sanjay Gandhi Postgraduate Institute of

Medical Sciences, Raebareli Road, Lucknow-U.P. 216014, India.

Email: anees892009@yahoo.in

doi:10.4021/wjnu25w
Keywords: Live related renal transplant; Percutaneous nephrectomy; Ureteral stenosis; Ureteral leak

\section{Introduction}

Renal transplantation is treatment of choice for end stage renal disease. Urological complications continue to be cause of graft dysfunction and patient death $[1,2]$. Urological complications used to be $29 \%$ in earlier series which came down to $5 \%$ in last two decades [2]. This change was attributed to preservation of periureteral fat, preserving fat between lower pole, renal vessels and ureter and new era immunosuppression regimen [3]. Krol et al determined surgical technique of vesicoureteral anastomosis as important factor in ureter related complications [4]. Ureteral complications of primary concern are ureteral stenosis and ureteral leak. Surgical technique during donor nephrectomy was of paramount importance as $70 \%$ of ureteral necrosis was present in distal ureter [5]. Vasculitis secondary to rejection episodes and vasoconstriction secondary to calcineurin inhibitor as immunosuppressants was also considered in etiology of ureteral stenosis [6]. Most of data for ureter related complications are from centres doing cadaveric transplantation [7]. With the introduction of laparoscopic live donor nephrectomy, there has been inherent fear of compromise to ureteral blood supply with impact on ureteral complications. This issue has not been addressed adequately in existing literature so far.

The purpose of this retrospective analysis was to find incidence of ureteral urinary leak and ureteral stenosis in live related renal transplantation and treatment offered for above mentioned complications. We also looked at the impact of live donor nephrectomy on these complications.

\section{Material and Methods}

We retrospectively evaluated incidence of ureteral complications and treatment offered in live related renal transplants (in phase 2 patients were studied prospectively with randomization). We also analyzed incidence of ureteral complica- 
Table 1. Overall Complications

\begin{tabular}{|c|c|c|c|c|}
\hline & stented & Non stented & Overall & Pvalue \\
\hline Ureteral leak & $0.4 \%(8 / 1757)$ & $6.1 \%(13 / 213)$ & $1.07 \%$ & $\mathrm{P}<0.05$ \\
\hline Ureteral stenosis & $0.6 \%(11 / 1757)$ & $2.3 \%(5 / 213)$ & $0.8 \%$ & $\mathrm{P}<0.08$ \\
\hline
\end{tabular}

tions in groups with and without stent especially to see effect of DJS (double J stent) in preventing urinary leak. Our technique of ureterovesical anastomosis (modified lich gregoir) has evolved in three phases. Phase 1 from 1989 to 1993 had 170 renal transplant recipients in which stent was placed as and when required (15/170). Phase 2 from January 1994 to April 1995 had 100 patients who were randomised to stent and no stent respectively (57 had stent and 43 had no stent). In phase 3 from May 1995 to December 2010 had 1757 patients and all were stented for 12 to 14 days except in select cases when stent was kept for longer period.

We routinely do extravesical modified lich gregoir ureterovesical anastomosis. Ureteral length was kept minimal. Sixteen cm 5 FR DJS was placed across ureterovesical anastomosis and stent was removed under local anaesthesia.

Donor nephrecomy was done by open method till 2002 and laparoscopic live donor nephrectomy was introduced after 2002. Routinely left kidney is harvested in laparoscopic procedure. Till now 800 laparoscopic live donor nephrectomise have been performed at our centre. The right donor nephrectomy is done predominantly by open technique. Per urethral catheter was kept for 5 days and negative suction drain was removed when daily drain output was less than 50 $\mathrm{mL}$. Patients were kept on anticholinergics (oxybutinin or tolterdine) till stent was in situ and antibiotics were given.

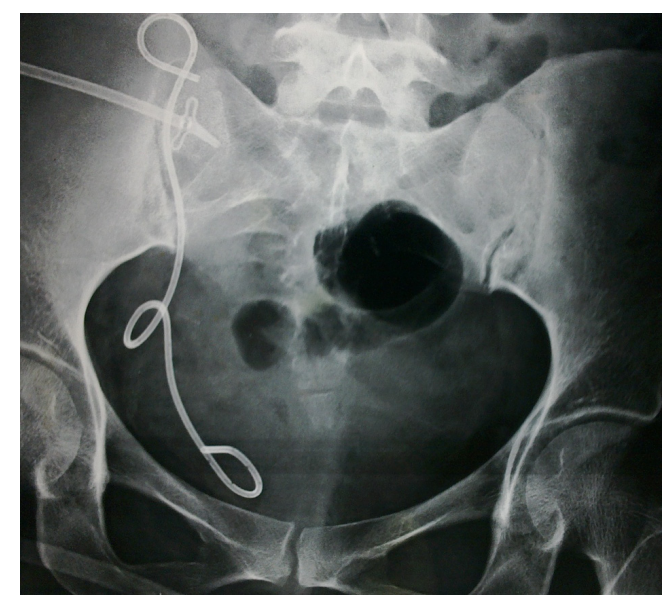

Figure 1. PCN done for hydronephrosis of transplant kidney despite stent in place.
All patients were kept on routine three drug immunosuppression (cyclosporine or tacrolimus, mycophenolate mofetil and steroids). Patients were monitored by serum creatinine, drain fluid creatinine, ultrasound abdomen, total WBC counts and urine culture.

Ultrasound was routinely done at day 1 and 7 and repeated after 3 months. Increase in serum creatinine also necessitated ultrasound. Hydronephrosis of recipient kidney was followed by renal scan. All events like urinary leak, obstructive hydronephrosis and UTI were recorded.

We evaluated ureteral complications and treatment offered for it. We also evaluated role of stent in preventing urine leak if any and incidence of ureteral complications in recipients of kidney harvested by laparoscopic method.

Statistical analysis was done to calculate mean and frequency using basic cross tabulation and descriptive analysis. Chi square test was used to calculate significance where needed. All analysis was done using SPSS version 17 software.

\section{Results}

In phase 1,15 out of 170 patients had stent because of some anticipation of problem at ureterovesical anastomosis. Elev-

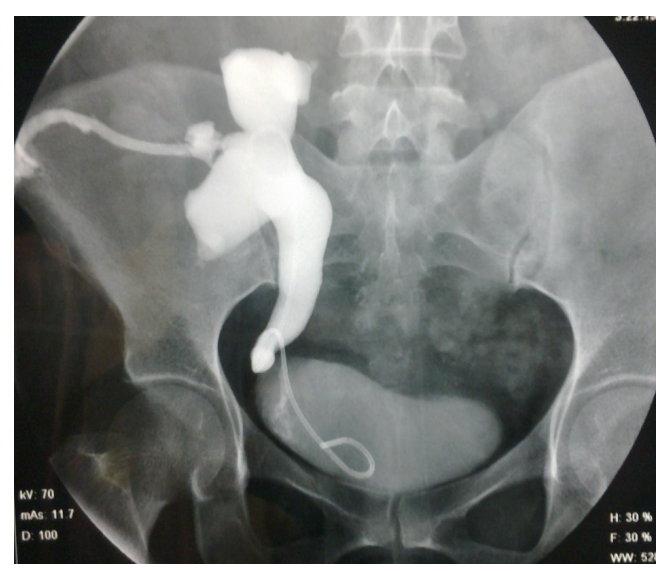

Figure 2. Nephrostogram in same patient reveal obstruction at the ureteroneocystostomy level, later cause was found out to be ureteral necrosis. 
Table 2. Treatment Options for Ureteral Leak

\begin{tabular}{ll}
\hline Treatment option & $\begin{array}{l}\% \text { (patients treated/total patients } \\
\text { with complications) }\end{array}$ \\
\hline Per urethral catheter & $61 \%(13 / 21)$ \\
PCN and antegrade DJS & $19 \%(4 / 21)$ \\
Open surgery & $23 \%(5 / 21)$ \\
\hline
\end{tabular}

en patients had ureteral leak (6.4\%). Ureteral obstruction was seen in 4 patients $(2.3 \%)$. In phase 2,57 patients were on stent and 43 patients were without stent. Demographic variables were comparable in both groups in terms of age, sex, donor age, associated illness, warm ischemia time and donor vascular anatomy. In group without stent, one had ureteral obstruction and two patients had leak. In group with stent no leak or stenosis was noted.

Till April 1995 (end of phase 2) overall incidence of ureteral leak was $6.1 \%(13 / 213)$ and ureteral stenosis was $2.3 \%$ $(5 / 213)$ in recipients without stent (Table. 1$)$.

All cases of urinary leak were initially treated with prolonged catheterization. Eight out of thirteen cases responded to catheterization alone. In one out of five cases PCN (percutaneous nephrostomy) was placed even with stent in place. He did not respond to endourological management and required surgical revision which revealed terminal necrosis of ureter which required revision of anastomosis (Fig. 1, 2). Four out of five revealed leak after stent was removed which responded to PCN placement. Two patients had forgotten stent which was removed 36 and 11 months respectively. No events like stent migration, broken stent, stent related stone, hematuria or stent related obstruction was noted.

From May 1995 onwards all vesicoureteral anastomosis was on stent and only eight cases of ureteral leak had been reported after that. Overall incidence of ureteral leak in stented group was $0.4 \%$ (8/1757). Two had ureteral necrosis as a cause of leak and one patient had an error of judgement as ureter was anastomosed with peritoneum. Five patients responded to PUC alone. Cases with ureteral necrosis were treated with revision ureteroneocystotomy as terminal ureter was necrosed. The 3 rd case, where ureter was anastomosed with peritoneum by mistake was identified as massive ascitis with increased drain output developed in immediate post op period. Biochemistry of ascitic fluid was suggestive of urine and exploration with ureteroneocystotomy was done.

Overall ureteral urinary leak was present in $0.4 \%$ of patients with DJS and in $6.1 \%$ of patients without stent in situ. This complication occurred after a mean of $4.6 \pm 2$ days of transplant. Per urethral catheterisation (PUC) was immediate treatment in our series. On failure of prolonged urethral catheter, PCN with antegrade stent was next step. In recipients without stent $8 / 13$ responded to PUC alone and $4 / 5$ responded to percutaneous nephrostomy (PCN) and antegrade double J stent (DJS) placement and one required ureteroneocystotomy for leak. In patients with stent $5 / 8$ responded to PUC alone and 3 required open surgical exploration (Table 2).

One interesting observation was that patients with stent in situ did not require PCN with antegrade DJS as treatment. They responded to PUC alone or surgical revision if terminal ureter had necrosis. All pts with leak that did not respond on PUC alone had leak at ureteroneocystostomy site. Necrosis in our series was present in terminal end.

Ureteral leak as a complication was more common before 2000 and is rarely seen in last decade. Out of 21 patients

Table 3. Treatment Options for Ureteral Stenosis

\begin{tabular}{ll}
\hline Treatment option & $\begin{array}{l}\% \quad \text { (patients treated/total patients with } \\
\text { complications) }\end{array}$ \\
\hline PCN and antegrade DJS & $69 \%(11 / 16)$ \\
Surgery (pyeloureterostomy or pyeloplasty) & $31 \%(5 / 16)$ \\
\hline
\end{tabular}


Table 4. Comparisons of Open and Laparoscopic Live Donor Nephrectomy

\begin{tabular}{llcc}
\hline & Laparoscopic donor nephrectomy & Open donor nephrectomy & P-value \\
\hline Urinary leak & $3 / 800(0.3 \%)$ & $18 / 1170(1.5 \%)$ & 0.12 \\
Ureteral stenosis & $6 / 800(0.75 \%)$ & $11 / 1170(0.9 \%)$ & 0.48 \\
\hline
\end{tabular}

with ureteral leak only three patients had kidney harvested laparoscopically $(0.3 \%)$ while in 18 cases open donor nephrectomy was done (1.5\%) (Table 3 ).

Ureteral stenosis was noted in $0.9 \%(16 / 1970)$ after mean of 40 days (14 - 170 days) of transplant from 1989 to 2010. Most commonly diagnosis was made on evaluation of rising serum creatinine when ultrasound showed hydronephrosis. Three out of 16 were diagnosed immediately after stent removal. All patients initially underwent PCN. After doing PCN antegrade nephrostogram revealed stenosis at ureterovesical junction in all and one patient had PUJ obstruction (Table 1).

Antegrade placement with 6/26 DJS was done in all patients with balloon dilatation. All but 2 patients required balloon dilatation. Both patients that did not required balloon dilatation were those who required $\mathrm{PCN}$ immediately after stent removal. As a policy stent was removed after 90 days. If serum creatinine showed rising trend after stent removal then patient planned for elective surgery after optimization with PCN. Five out of 16 pts required surgical revision as PCN with antegrade stent did not work in these patients. Native pyeloureterostomy was done in all. One patient had pelviureteric junction obstruction (Table 4).

In 16 patients with ureteral stenosis 11 patients had kidney removed from open method $(0.9 \%)$ and six from laparoscopic method $(0.75 \%)$ (Table 3$)$.

\section{Discussion}

Ureter related complications continue to be an important cause of graft dysfunction and patient death. Various series have addressed this pertinent issue. Endourological and surgical management of stenosis and leak are widely discussed in existing literature. Impact of laparoscopic donor nephrectomy on ureter related complications remains a dilemma. Our series has addressed all these pertinent issues including role of stented ureterovesical anastomosis.

Zavos et al analysed urological complications in 1525 patients which included cadaveric and live related renal transplant and routinely did stented lich gregoir anastomosis [8]. Ureteral stenosis was present in $4.4 \%$ patients. Various causes of stenosis were clot, kink, torsion, stone, necrosis, lymphocele and warm ischemic stricture [8]. They have used native to graft ureteroureterostomy (end or side) also as treatment option. Nine out of ten patients had normal graft function after corrective measures were taken.

Sansalone et al similarly analyzed ureter related compliations and preventive role of stent in 15 years of transplant experience [3]. Ureteral stenosis was present in $1.5 \%$ of patients with stent and in $1.2 \%$ patients without stent ( $\mathrm{P}$ value not significant). They also used boari flap selectively in open surgical procedure to treat stenosis.

Ureteral obstruction during 1st month was not common and was commonly due to clot or technical errors. Ureteral obstruction after 1st month was due to ureteral stenosis [9]. Late ureteral stenosis has been discussed by Fontanna et al [10]. Incidence of late ureteral stenosis in that series was $3.16 \%$ and they also concluded that donor age greater than 65 years, two renal vessels and delayed graft function as risk factor for late warm ischemic ureteral stenosis.

Juaneda et al had a series of 56 patients of ureteral stenosis treated with percutaneous balloon dilatation with $45 \%$ success rate [11].

Nitinol self expanding metallic ureteric stents has been tried in patients with high surgical risk with reasonable success [12].

Overall incidence of ureteral stenosis in our series was $0.8 \%$ with $2.3 \%$ in patients without stent and $0.6 \%$ in patient with stent, $69 \%$ responded to antegrade stent and $31 \%$ required surgical revision.

Urinary leak was present in $1.6 \%$ of patients of Zavos series [8]. Urinary leak in patients with stent were $0.3 \%$ as compared to $2.6 \%$ in non stented patients $(\mathrm{P}<0.005)$ in series described by sansolone et al.

Guleria et al in a series of 507 patients with 507 unsplinted Leadbetter Politano anastomosis has ureteric complication rate of $7.7 \%$ which decreased to $3.8 \%$ with lich gregoir anastomosis was with stent (total 1186 patients). In above series percutaneous radiological techniques were used to salvage majority of complications (84.7\%) [13].

An interesting observation made by giakoustidwas et al in his study where he compared outcome of two groups stent versus no stent found that though stent did not significantly decreased urine leak but gravity of urine leak in stented group was less severe as they did not required surgical intervention 
for leaks [14]. In our study also patients with leak having stent responded to urethral catheterization alone in majority.

Overall incidence of ureteral leak in our study was $1.07 \%$ with $6.1 \%$ in patients without stent and $0.4 \%$ in patients with stent. $61 \%$ patients with leak responded to perurethral catheterization alone, $19 \%$ responded to stent and $23 \%$ responded to open surgery. Decreased dose of steroids has reduced incidence of ureteric complications in last 20 years [2].

Argument usually put forward against use of routine stent are increased risk of storage LUTS,UTI, hematuria, stone formation, stent encrustation, broken stent, stent migration, forgotten stent and extra cost incurred during stent removal. Some authors argue that good vascularity and sound surgical technique was good substitute for routine use of stent in ureterovesical anastomosis [15].

Numerous studies have shown that stent with antibiotics was not associated with increased incidence of UTI. One study in which stent was kept for 14 days has shown that complication associated with long term stent placement like stone formation, encrustation, migration, forgotten stent, UTI, storage LUTS can be easily dealt with. Stent for 14 days with prophylactic cotrimoxazole was financially more viable than dealing with prolonged hospitalization secondary to stent related complications. Moreover small urinary leaks and necrosis was taken care by stent for two weeks. No study has shown increased incidence of hematuria secondary to stent [16].

Cautious dissection of golden triangle, maintaining peiureteral fat and adventitial tissue of ureter, splinted anterior lich gregoir anastomosis, using minimal ureteral length and decreasing use of steroids are factors under surgical control to decrease incidence of ureter realted complications like stenosis and leak $[1,9,16]$

In our study regarding ureteral leak there seems to be an aberration as leak was more common in patients whose kidney was removed by open method. This aberration is explained by fact that ureteral leak was more common before 2000 probably due to high dose of steroids used in those days as compared to present era and we used to do open donor nephrectomy before 2000 .

Drawback of our study is that it is retrospective and duration of study is long (20 years). During this period immunosuppression regimens have changed and surgical technique got refined and better with time and experience.

\section{Conclusions}

Ureteral stenosis and leak are two major complications. Whereas ureteral leak responded to either conservative or Endourological management in majority of cases, management of ureteral stenosis required open surgical intervention more often.

Routine use of DJ stent appears to help in prevention of ureteral leak but doesn't have any impact on ureteral steno- sis.

Laparoscopic live donor nephrectomy doesn't seem to have any adverse effect on ureteral blood supply.

\section{Conflict of Interest}

Nil.

\section{References}

1. Butterworth PC, Horsburgh T, Veitch PS, Bell PR, Nicholson ML. Urological complications in renal transplantation: impact of a change of technique. Br J Urol. 1997;79(4):499-502.

2. Streeter EH, Little DM, Cranston DW, Morris PJ. The urological complications of renal transplantation: a series of 1535 patients. BJU Int. 2002;90(7):627-634.

3. Sansalone CV, Maione G, Aseni P, Mangoni I, Soldano S, Minetti E, Radaelli L, et al. Advantages of short-time ureteric stenting for prevention of urological complications in kidney transplantation: an 18-year experience. Transplant Proc. 2005;37(6):2511-2515.

4. Krol R, Ziaja J, Chudek J, Heitzman M, Pawlicki J, Wiecek A, Cierpka L. Surgical treatment of urological complications after kidney transplantation. Transplant Proc. 2006;38(1):127-130.

5. Keller H, Noldge G, Wilms H, Kirste G. Incidence, diagnosis, and treatment of ureteric stenosis in 1298 renal transplant patients. Transpl Int. 1994;7(4):253-257.

6. Streem SB, Novick AC, Steinmuller DR, et al: Long term efficacy of ureteral dilatation for transplant ureteral stenosis. J Urol 1998; 140:32

7. Shoskes DA, Hanbury D, Cranston D, Morris PJ. Urological complications in 1,000 consecutive renal transplant recipients. J Urol. 1995;153(1):18-21.

8. Zavos G, Pappas P, Karatzas T, Karidis NP, Bokos J, Stravodimos K, Theodoropoulou E, et al. Urological complications: analysis and management of 1525 consecutive renal transplantations. Transplant Proc. 2008;40(5):1386-1390.

9. Faenza A, Nardo B, Catena F, Scolari MP, d'Arcangelo GL, Buscaroli A, Rossi C, et al. Ureteral stenosis after kidney transplantation. A study on 869 consecutive transplants. Transpl Int. 1999;12(5):334-340.

10. Fontana I, Bertocchi M, Rossi AM, Gasloli G, Santori G, Barabani C, Fregatti P, et al. Late ureteral stenosis after kidney transplantation: a single-center experience. Transplant Proc. 2010;42(4):1174-1175.

11. Giakoustidis D, Diplaris K, Antoniadis N, Papagianis A, Ouzounidis N, Fouzas I, Vrochides D, et al. Impact of double-j ureteric stent in kidney transplantation: singlecenter experience. Transplant Proc. 2008;40(9):3173- 
3175.

12. Burgos FJ, Pascual J, Marcen R, Garcia-Navas R, Garcia IG, Alarcon C, Gomez V, et al. Self-expanding metallic ureteral stents for treatment of ureteral stenosis after kidney transplantation. Transplant Proc. 2005;37(9):38283829.

13. Guleria S, Chahal R, Madaan S, Irving HC, Newstead CG, Pollard SG, Lodge JP. Ureteric complications of renal transplantation: the impact of the double J stent and the anterior extravesical ureteroneocystostomy. Transplant Proc. 2005;37(2):1054-1056.
14. Juaneda B, Alcaraz A, Bujons A, Guirado L, Diaz JM, Marti J, de la Torre P, et al. Endourological management is better in early-onset ureteral stenosis in kidney transplantation. Transplant Proc. 2005;37(9):3825-3827.

15. Rajaian S, Kumar S. There is no need to stent the ureterovesical anastomosis in live renal transplants. Indian J Urol. 2010;26(3):454-456.

16. Agarwal G, Palagiri AV, Bouillier JA, Cummings JM. Endoscopic management of ureteral complications following renal transplantation. Transplant Proc. 2006;38(9):2921-2922. 